\title{
Ästhetisches Lernen im DaF-Unterricht: Musik - Kunst - Film - Theater - Literatur
}

\author{
Bericht zum DaF-Kongress vom 11. bis 13. März 2013 an der \\ Universidad Nacional Autónoma de México (UNAM) in \\ Mexiko-Stadt
}

\section{Nils Bernstein und Charlotte Lerchner mit Unterstützung von Julia Collazo, Paul Schneeberger und Jeruna Tiemann}

Vom 11. bis zum 13. März 2013 fand der Kongress „Ästhetisches Lernen im DaF-Unterricht. Musik - Kunst - Film - Theater - Literatur" an der Universidad Nacional Autónoma de México (UNAM) in Mexiko-Stadt statt. Organisiert wurde diese Veranstaltung in Zusammenarbeit mit dem Fremdsprachenzentrum (Centro de Enseñanza de Lenguas Extranjeras - CELE) der selbigen Universität, dem Goethe-Institut Mexiko, dem Deutschen Akademischen Austauschdienst (DAAD), dem Österreichischen Austauschdienst (OeAD), dem Österreichischen Kulturforum Mexiko sowie dem mexikanischen Deutschlehrerverband (AMPAL).

Ziel des Kongresses war es, die verschiedenen Kunstformen unter Beachtung ihrer jeweils eigenen Ästhetik in den Mittelpunkt zu stellen. Zwei zentrale Fragestellungen leiteten dabei die Diskussion: Zum einen wurde die Rolle der Künste im heutigen DaF-Unterricht hinterfragt. Zum anderen wurde erörtert, wie man das ästhetische Lernen vor dem Hintergrund der zunehmenden Kompetenzorientierung stärken kann.

An dieser Stelle setzte der Eröffnungsvortrag zum Thema, Ästhetische Bildung im fremdsprachlichen Literaturunterricht als Grundlage für nachhaltiges Lernen

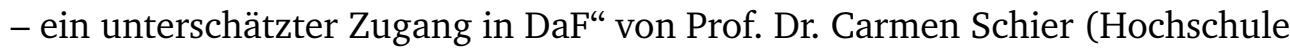
für angewandte Wissenschaften Coburg) an. Sie verwies darauf, dass sowohl sinnliche Wahrnehmung als auch Emotionen in der heutigen Bildung kaum eine Rolle spielten, obwohl ästhetisches Lernen vielfältige Kompetenzen wie Kreativität, emotionale Intelligenz und flexibles Denken fördere und Nachhaltigkeit erzeugen könne. Am Beispiel des Lesens und anhand von Erhebungen zu Lese-Typen zeigte sie, wie das Lesen auf ein rein funktionales Verstehen reduziert wird, sodass seiner Bedeutung für Fremdverstehen und persönliche Entwicklung keine Beachtung geschenkt wird.

In der Sektion Literatur hielt Dr. Dietrich Rall (UNAM) einen Semiplenarvortrag zum Thema „Die Farbe Blau: Literatur und Kunst im DaF-Unterricht“. Hierbei bildete „Die Farbe Blau” als ästhetisch, literaturhistorisch und komparatistisch bedeutendes Thema den Ausgangspunkt für eine interdisziplinär und 
interkulturell angelegte Unterrichtseinheit, die sich vornehmlich an eine universitäre Zielgruppe richtete. Nach farbtheoretischen und farbpsychologischen Ausführungen veranschaulichte Rall, wie die Farbe Blau als Projektarbeit im DaF-Unterricht ästhetisches Lernen ermöglicht.

In seinem Vortrag „[U+02CE] o so viel vieh`. Lyrik im DaF-Unterricht - Instrumentalisierung vs. Spaß an der Freude" ging Dr. Nils Bernstein (DAAD-Lektor an der UNAM) der Frage nach, inwieweit literarische Texte im Allgemeinen und Lyrik im Besonderen zur Vermittlung sprachlicher und landeskundlicher Inhalte verwendet werden können. Dabei erörterte er zunächst, aus welchem Grund Lyrik geschrieben und gelesen wird und stellte hierzu metalyrische Kommentare und literaturwissenschaftliche Kommentare gegenüber. Schließlich kam er zum Resümee, dass einerseits jede sprachliche Handlung im Fremdsprachenunterricht als Instrumentalisierung insinuiert werden könne. Andererseits würde bei der Behandlung von Gedichten egal mit welchem Lernziel - die Literarizität der Texte nicht vergessen. Mit anderen Worten: Gedichte dürfen durchaus verwendet werden, um Phonetik, Wortschatz oder Landeskunde zu vermitteln.

Der ästhetischen Bildung mit Schwerpunkt auf dramapädagogischen Ansätzen verpflichtete sich Dr. Manfred Schewe (University College Cork, Irland) in seinem Semiplenarvortrag „Perspektiven einer neuen performativen Lehr- und Lernkultur im DaF-Unterricht“. Er legte dar, dass „performative Fremdsprachenpraxis" kulturgeschichtlich fest verankert sei. Eindrücklich und anschaulich belegte er die Rolle der ästhetischen Bildung als Tradierung von Träumen und Hoffnungen sowie symbolisch vermittelter Welterkenntnis anhand einer Analyse der bekannten, 1967 von Leo Lionni veröffentlichen Bilderbuchgeschichte „Frederick“. Anschließend skizzierte Schewe die Forschungsgeschichte der Dramapädagogik im Fremdsprachenunterricht. Trotz der festen kulturgeschichtlichen Verankerung der Theaterkunst als Inspirationsquelle für Sprachlehrende, setzte diese erst in den 1990er Jahren an. Inzwischen aber seien dramapädagogische Ansätze im Fremdsprachenunterricht unverzichtbar, da man mit ihnen den Anforderungen lebenslangen Lernens gerecht werde und u.a. das Vorstellungsvermögen der Lernenden, das Denken in Bildern und szenischen Arrangements optimal anrege. ${ }^{1}$

In beispielhafter Weise anwendbar waren die didaktisch versierten Vorschläge des Theaterworkshops der Wiener Improvisationstheatergruppe artig. AnneMarie Kuhfuß und Lino Kleingarn zeigten in zwei parallelen Gruppen zahlreiche Übungen: von Kennenlernspielen und Auflockerungsübungen über Kurzszenen für Sprachanfängerinnen und -anfänger bis hin zu elaborierten Dialogszenen für höhere Sprachniveaus. Als theoretische Grundlage des Konzepts von artig dient das Improvisationstheater nach Keith Johnstone. Drei Regeln sind dabei Voraussetzung für die Umsetzung dieses Ansatzes im Fremdsprachenunterricht: Erstens, die erste Idee, die einem in den Kopf kommt, zählt. Zweitens, man muss affirmativ sein und einfach ja sagen, Fehler gibt es nicht. Und drittens,

\footnotetext{
${ }^{1}$ Vgl. in diesem Kontext auch den Artikel Taking Stock and Looking Ahead: Drama Pedagogy as a Gateway to Performative Teaching and Learning in dieser Ausgabe.
} 
Scheitern sollte als Möglichkeit zum nochmaligen Versuch verstanden werden.

Auf eine wichtige Grundlage für die Sektion Musik ging Matthias Perner (Fachsprachenzentrum der Universität Hannover) bereits in seinem Semiplenarvortrag „Musik im DaF-Unterricht: Von der Sprache zur Musik und zurück“ ein, indem er das notwendigerweise vorhandene, nicht aber allen geläufige Beziehungsgeflecht zwischen Sprache und Musik beleuchtete. Neben einem geschichtlichen Überblick über den Einsatz von Musik im Unterricht stellte er Funktionen von Musik im DaF-Unterricht dar und verwies hier besonders auf die Möglichkeit zur Vermittlung von Sprachrhythmus und Sprachmelodie. Dies veranschaulichte er durch verschiedene Lehr-Lernarrangements, in denen er selbst mit RaP gearbeitet hatte. Genauer ging er auf diese praktische Arbeit in seinem Workshop „DaF \& RaP [U+02BB] War ich das? [U+02BC] “ ein.

Sowohl theoretisch fundiert als auch anwendungsbezogen war der Vortrag „Übersetzen von Liedern im DaF-Unterricht“ von Ulrike Pleß (DAAD-Lektorin an der Universidad de Guadalajara, Mexiko). Nach einer kritischen Einführung zur Relevanz des Übersetzens im DaF-Unterricht stellte sie Arbeiten von Studierenden vor, die Liedtexte selbstständig von der Zielsprache Deutsch in die Muttersprache Spanisch übersetzt hatten. Diese intensive Auseinandersetzung mit bekannten deutschen Liedtexten führte nicht nur dazu, dass sich die Studierenden mit landeskundlichen Hintergrundinformationen befassten, sondern erlaubte außerdem eine effektive Wortschatzarbeit.

In der Sektion Kunst konnten die Teilnehmenden im Workshop „Bilder tanzen im Kopf. Workshop zur kreativen Sprachproduktion anhand von Kunstwerken“von Renate Köhl-Kuhn (Goethe-Institut München) die vielfältigen unterrichtlichen Einsatzmöglichkeiten von Kunst in ihren Ausdrucksformen Malerei, Plastik und Fotografie erleben. In einem Galeriespaziergang lernten die Teilnehmenden unterschiedliche Übungsformen kennen, in denen Kunstwerke als Sprechimpulse für die kreative Textproduktion dienen und Interaktion anregen. Deutlich wurde dabei das Potenzial dieser Methoden für die Schulung ästhetischer Kompetenzen im DaF-Unterricht. Des Weiteren wurde gezeigt, wie Bildende Kunst mit anderen Künsten wie Theater und Literatur verknüpft werden kann. Die gemeinsame Reflexion über die mit den Methoden verbundenen Lernziele, über mögliche Lernergruppen und angesprochene Kompetenzbereiche bereitete den Weg für die praktische Umsetzung im DaF-Unterricht.

Auf ähnliche Weise erschlossen sich die Teilnehmenden im Workshop "Österreich. Malerisch" von Dr. Walter Thalhammer (Universität Wien) die vielfältigen Einsatzmöglichkeiten von Malerei. Anhand von Selbstportraits, Landschafts- und Szenenbildern österreichischer Künstler wie Klimt, Schiele und Kokoschka wurden Übungen zum Training der Sprech-, Hör- und Schreibfertigkeit ausprobiert und reflektiert. Entsprechend des Anspruchs, ästhetische Kompetenzen zu schulen, spiegelte die Auswahl der Bilder ein breites Kunstverständnis wider, das abstrakte Bilder genauso einschloss wie die leichter zugänglichen, gegenständlichen Bilder.

Die Sektion Film wurde mit dem Semiplenarvortrag „Film und DaF-Unterricht“ 
von Dr. Tina Welke (Universität Wien) eingeleitet. Die Vortragende ging zunächst von Film als einer audio-visuellen Komposition aus, deren einzelne Elemente (im Wesentlichen Bild, Ton und Text) man im DaF-Unterricht nutzen und einsetzen kann. Gesteigert werden könne die Wirkung des Mediums zum einen durch die Vermittlung von Medienkompetenz und zum anderen durch einen methodischen Zugang, der die Arbeit mit dem Medium in VOR, WÄHREND und NACH dem Sehen gliedere. Außerdem führte sie das Hör-Seh-Verstehen als fünfte Fertigkeit ein und wies auf die Bedeutung des Mediums für die Landeskunde hin.

Verónica Cedeño Mora (Deutschlehrerin am Sprachenzentrum der UNAM) und Patricia Ángeles Delgado (Italienischlehrerin am Sprachenzentrum der UNAM) stellten in ihrem Vortrag „Interkulturalität durch Filme im Deutschunterricht“ ihre Ergebnisse aus dem umfangreichen Projekt „Partidos en 3: Un encuentro entre alemanes, italianos y ... méxicanos“ („Durch drei geteilt: Ein Treffen zwischen Deutschen, Italienern und ...Mexikanern") vor. Ziel dieses Projektes war es, mit mexikanischen Deutsch- und Italienischlernenden Ursachen und Folgen von Stereotypisierung anhand von Filmbeispielen zu reflektieren. Durch die Diskussion über die Filme sowie die Erstellung einer Collage sollten Stereotypen, verstanden als Komplexitätsreduktionen, genauer differenziert werden.

Dass die Position der verschiedenen Künste im Fremdsprachenunterricht sehr unterschiedlich ist, zeigte sich bei der Diskussion im Abschlussplenum. Sowohl die theoretische Fundierung betreffend als auch in unterrichtspraktischer Hinsicht unterscheiden sie sich. Gemeinsam ist ihnen aber, dass sie ihren Einsatz im Unterricht rechtfertigen müssen. Der Kongress hat einen wichtigen Anstoß in diese Richtung gegeben. Dennoch ist noch viel notwendig, um die Stellung des ästhetischen Lernens zu verbessern. Nicht nur inhaltlich wurde der Kongress von vielen Seiten gelobt. Hervorgehoben wurde auch die Bedeutung der multilateralen Zusammenarbeit. Um Deutsch als Fremdsprache in Mexiko zu fördern, sind weitere Kooperationen dieser Art unbedingt wünschenswert.

Weitere Informationen zum Kongress finden Sie auf der Internetseite: http://www.cele. unam.mx/dafkongress (Stand: 20. April 2013). 